\title{
Magnetic field of the transition current system: dawn-dusk asymmetry
}

\author{
E. S. Belenkaya ${ }^{1}$, I. I. Alexeev ${ }^{1}$, and C. R. Clauer, Jr. ${ }^{2}$ \\ ${ }^{1}$ Skobeltsyn Institute of Nuclear Physics, Moscow State University, Moscow 119992, Russia \\ ${ }^{2}$ Virginia Polytechnic Institute and State University Bradley Department of Electrical and Computer Engineering, Blacksburg, \\ VA 24061, USA
}

Received: 29 August 2006 - Revised: 23 July 2007 - Accepted: 3 August 2007 - Published: 29 August 2007

\begin{abstract}
In this paper we consider the interactions of coronal mass ejections (CMEs) with the Earth's magnetosphere for the specific case in which there is a sharp increase in the dynamic pressure (interplanetary shock) that is associated with a simultaneous northward turning of the interplanetary magnetic field (IMF) from the near horizontal direction. Previously, we have shown that under such circumstances, the so-called transition current systems arise. These temporary high-latitude current systems create a low-latitude asymmetric magnetic field on the ground with a large northward field enhancement on the nightside and little or no field increase near local noon. Here we investigate the dawn-dusk asymmetry of the low-latitude on-ground magnetic field of the transition current system caused by the IMF. Analysis of the Region 1 current circuit for northward IMF shows a change in its shape controlled by different IMF components. Due to this geometrical effect, the maximum and minimum magnetic field disturbances appear to be shifted. The obtained results supplement and define more precisely the locations of the magnetic disturbance extrema retrieved recently by Clauer et al. (2001). The results of this study are compared with the available observations. A good accordance is demonstrated.
\end{abstract}

Keywords. Magnetospheric physics (Current systems; Magnetosphere-ionosphere interactions; Magnetospheric configuration and dynamics)

\section{Introduction}

A typical sudden compression of the magnetosphere, caused by the encounter with the coronal mass ejection (CME), is associated with a world-wide enhancement of the northward ground magnetic field at low-latitudes with a slightly larger

Correspondence to: E. S. Belenkaya

(elena@dec1.sinp.msu.ru) enhancement on the dayside. Clauer et al. (2001) introduced the concept of a three-dimensional transition current system that exists for some characteristic time in the polar regions and is caused by the rotation of the interplanetary magnetic field (IMF) from the near horizontal direction to the northward direction simultaneous with the passage of an interplanetary shock. It was shown by Clauer et al. (2001) that under these conditions in the solar wind, the three-dimensional transition current system creates at low latitudes the ground magnetic field perturbation which explains an observed asymmetric increase in the $H$ or $X$ component of the geomagnetic field northward on the nightside and much weaker, or even slightly southward at local magnetic noon. The analysis of the dependence of the intensity and direction of the field-aligned current in the transition current system to the radial $\left(B_{x}\right)$ and azimuthal $\left(B_{y}\right)$ components of the IMF and to the high-latitude ionospheric conductivity has been done by Belenkaya et al. (2004). Here we investigate the dawn-dusk asymmetry of the low-latitude magnetic field of the transition current system caused by the IMF components. Results of this study are compared with available observations.

\section{Short description of the transition current system}

For self-consistency we briefly describe here the transition current system model. It was shown (Clauer et al., 2001; Belenkaya et al., 2004) that the three-dimensional transition current system should form during a characteristic time period of $t_{r} \sim 30-40 \mathrm{~min}$ after the magnetosphere encounters a CME-related shock containing a change from horizontal to northward IMF. This system includes the field-aligned NBZcurrents, the ionospheric Pedersen currents in the region of open field lines in the polar caps, and the field-aligned currents concentrated at the ionospheric open-closed field line boundaries ( $\theta=\theta_{m}$, where $\theta$ is a co-latitude). These boundary

Published by Copernicus Publications on behalf of the European Geosciences Union. 


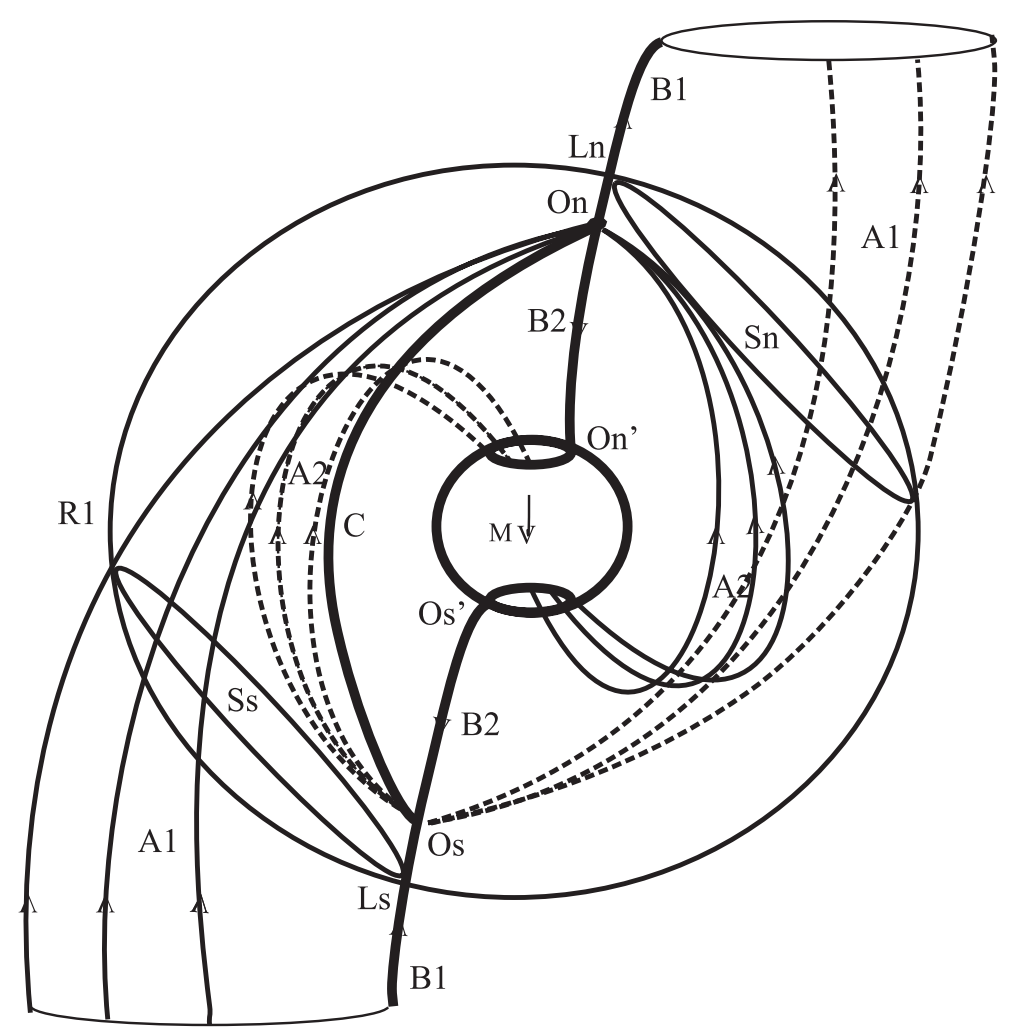

Fig. 1. Magnetospheric magnetic field structure for northward IMF. Sphere of the radius $R_{1}$ schematically marks the magnetopause. $A 1$ and $A 2$ are the boundaries between the field lines of different topological types. $B 1$ and $B 2$ are the singular magnetic field lines perpendicular at the neutral points $O n$ and $O s$ to the magnetic field line surfaces $A 1 O n A 2$ and $A 1 O s A 2 . O n^{\prime}$ and $O s^{\prime}$ are the ionospheric projections of the magnetic field neutral points. $C$ is the separator line. $M$ is the Earth's dipole. $S_{S}$ and $S_{n}$ are the southern and northern sections of the magnetopause by the open field line tubes. $L_{S}$ and $L_{n}$ are the points of the intersections of $S_{S}$ and $S_{n}$ with the singular magnetic field lines $B_{1}$, respectively.

field-aligned currents may be interpreted as the Region 1 currents.

For northward IMF two magnetic field neutral points arise inside the magnetosphere, in the cusp regions (e.g. Belenkaya, 1998a). Figure 1 shows the magnetic field structure for the northward IMF. The magnetopause, for simplicity, is schematically shown as a sphere of radius $R_{1} ; S_{n}$ and $S_{s}$ are its sections by the northern and southern open field line tubes, respectively; $O_{n}$ and $O_{s}$ are the northern and southern magnetic nulls. The boundary surfaces $A_{1}$ and $A_{2}$ dividing the magnetic field lines of different topological types (separatrix surfaces) are connected at the separator line $C$ (Siscoe et al., 2001; Blomberg et al., 2005).

$A_{1}$ and $A_{2}$ are magnetic field line surfaces intersecting at the neutral points. $B_{1}$ and $B_{2}$ are singular magnetic field lines (named by Siscoe et al., 2001 as "stemlines") perpendicular to these surfaces at magnetic nulls. Field lines $A_{1}$ form a boundary of the open field line tube. As the whole surface $A_{1}$ converges to (or diverges from) a neutral point, field lines intersecting magnetic null go through a diffusion region. As a result, in particular, strong field-aligned currents arise along a boundary of the open field line tube. From Fig. 1 it is seen that along the singular field line $B_{2}$, these strong field-aligned currents flow into and out of the ionosphere in the vicinity of the cusp projection; these are the field-aligned cusp currents arising during northward IMF, or NBZ currents. Thus, the field-aligned NBZ currents are directly driven by the MHD solar wind generator (Belenkaya, 2002).

Field lines of the surface $A_{2}$ connect the neutral point with the ionospheric open field line boundary of the opposite hemisphere. Thus, for the northward IMF, the open field line ionospheric boundary is an equipotential (Belenkaya, 1998b). The convection lines (which are lines of constant electric potential) do not intersect it, contrary to the southward IMF case, and form the vortex structure inside the polar cap converging to the cusp projection. So, the convection during the characteristic time period $t_{r}$ is mainly contained within the polar cap (the development of convection on the closed field lines will occur after $t_{r}$ (Clauer et al., 2001)). Characteristic time $t_{r}$ equals $L_{x} / V_{x} \sim 30-40 \mathrm{~min}$, where $L_{x}$ is the mean length of the open field line tube section at the magnetopause along the $x$ direction, and $V_{x}$ is the solar wind velocity. During this time period, the three-dimensional current system corresponding to northward IMF develops only 
partially. Only the current system directly driven by the MHD solar wind generator (transition current system) forms. This current system is mainly connected with the open field lines. The penetration of convection on the closed field lines occurs indirectly (contrary to the case of southward IMF), only through the region of the ionospheric cusp projection, where convection vortices intersect. So, during the first 30 $40 \mathrm{~min}$, the ionospheric closure currents correspond to the case where the "effective low-latitude ionospheric conductivity" is equal to zero. In this case, for $t<t_{r}$, Fukushima's Theorem (Fukushima, 1969) does not apply, and the Pedersen current magnetic field does not cancel the field-aligned current field measured at a ground station, so the magnetic field of the transition current system can be observed by the on-ground magnetometers.

As during $t<t_{r}$ the convection is contained within the polar cap, the ionospheric Pedersen currents caused by the NBZ currents cannot penetrate into the ionospheric closed field line region, and consequently, should be closed by the fieldaligned Region 1 currents at the polar cap boundary. Thus, we can say that, finally, in the transition current system, the Region 1 currents are driven by the NBZ currents.

Analytical calculations (Clauer et al., 2001) allowed us to obtain the magnetic field, caused by the transition current system at noon and midnight on the Earth's equator $\left(B_{t c s-z}\right)$ :

$B_{t c s-z} \approx \mp 1.3 B_{J} \sin \theta_{m}$,

where

$B_{J}=\left(\mu_{0} J\right) /\left(2 \pi R_{E}\right)$,

here $J$ is the strength of the field-aligned current in the transition current system, and $R_{E}$ is the Earth's radius; minus sign (plus) corresponds to noon (midnight), and the minus sign represents a southward field. $J$ was estimated from the approximate formula

$J=\Sigma_{P}\left|\delta \Phi_{n p c}\right|$,

where $\Sigma_{P}$ is the ionospheric Pedersen conductance, and $\delta \Phi_{n p c}$ is the polar cap electric potential drop. The derivation of Egs. (1-3) are found in Clauer et al. (2001). It was shown by Alexeev et al. (2007) ${ }^{1}$ that for northward IMF, $\delta \Phi_{n p c}$, and consequently $J$ (see Eq. 3 ), are proportional to $B_{z}$ and $V^{1 / 2}$, and very weakly depends on $n\left(B_{z}, V\right.$, and $n$ are the solar wind magnetic field northward component, velocity, and density, respectively). Thus, the field-aligned current strength in the transition current system generated by the IMF northward rotation depends on the solar wind parameters, and in particular, increases with increasing of velocity. For this reason, $J$ should correlate with the solar wind dynamic pressure. When CME brings the pressure jump simultaneous with the northward IMF rotation, a strong transition

\footnotetext{
${ }^{1}$ Alexeev, I. I., Belenkaya, E. S., Bobrovnikov, S. Y., and Kalegaev, V. V., Cumnock, J. A., and Blomberg, L. G.: Magnetopause mapping to the ionosphere for northward IMF, Ann. Geophys., submitted, 2007.
}

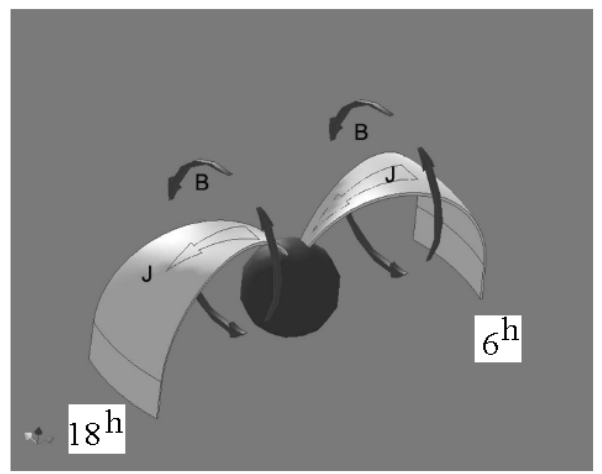

Fig. 2. Magnetic field $\mathbf{B}_{\text {tes }}$ caused by the Region 1 current sheets, $J$, flowing into the ionosphere in dawn and flowing out of the ionosphere in dusk. The field $\boldsymbol{B}_{t c s}$ is southward at noon and northward at midnight.

current system develops. Moreover, usually, there is an enhanced magnetic field in the CME also, which additionally amplifies $\delta \Phi_{n p c}$ and $J$. This is why, in such cases, the magnetic field of the transition current system becomes strong enough to be measured by the on-ground magnetometers.

The inward and outward NBZ currents are placed close to each other in the high-latitude ionosphere (the distance between their footprints is proposed to be of the order of the latitudinal scale of the ionospheric cusp region, $\delta \theta \sim 1^{\circ} \approx 112 \mathrm{~km}$ ). The contribution of these two antiparallel field-aligned currents to the magnetic field at the Earth's equator is proportional to $\sin \delta \theta$. The corresponding contribution of the Region 1 currents is proportional to $\sin \theta_{m}$. Assuming $\theta_{m} \sim 17^{\circ}$, we estimate the ratio of the magnetic fields of the NBZ and Region 1 currents to be equal to the ratio of $\sin \delta \theta$ to $\sin \theta_{m}$. Thus, the magnetic effect of the NBZ currents at the Earth's equator is $\sim 0.06$ of the magnetic field of the Region 1 field-aligned currents (Clauer et al., 2001). For this reason, in estimating of the effect of the transition current system at the Earth's equator, the NBZ field-aligned currents contribution can be omitted (however, the NBZ currents themselves play a significant role in supporting the Region 1 currents in the transition current system). It should be noted that the NBZ currents located near the cusp and existing during the northward IMF differ from the so-called cusp region 0 field-aligned currents. Figure 2 shows the magnetic field of the Region 1 currents which is southward in the dayside and northward in the nightside.

Clauer et al. (2001) calculated the magnetic field of the transition current system at the Earth's equator at noon and midnight suggesting that the field-aligned currents at the open-closed field line boundary may be represented by two currents flowing to the ionosphere at dawn and out of the ionosphere at dusk. In the paper by Clauer et al. (2001), the effects of the $B_{x}$ and $B_{y}$ IMF components were not taken into account. 


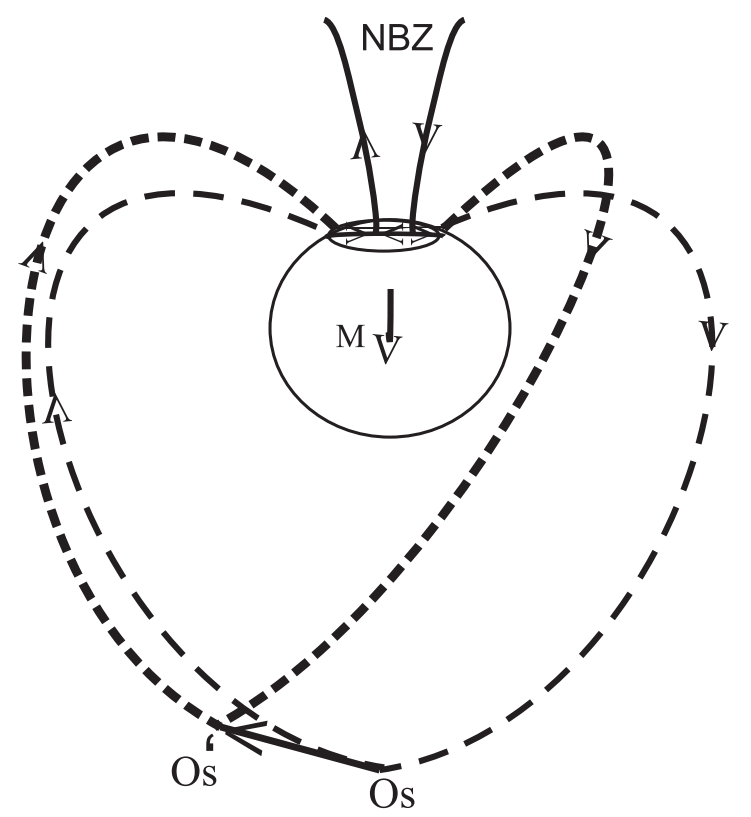

Fig. 3. Scheme of the northern transition current system. textbfM is the Earth's dipole. NBZ currents flowing into the ionosphere in the dusk, flow then on the ionosphere as the Pedersen currents, which are closed later by the field-aligned Region 1 currents flowing out from the northern ionospheric open field line boundary (marked by a circle) to the southern neutral point ( $O_{s}$ for the IMF with $B_{x}=0$, $B_{y}=0$, and $B_{z}>0$, and $O_{s}^{\prime}$ for the IMF with $B_{x} \neq 0, B_{y} \neq 0$, and $B_{z}>0$ ). Then they flow from the southern neutral point along field lines going to the dawn open field line boundary of the northern polar cap. After that they flow as the Pedersen ionospheric currents, and later are closed by the NBZ field-aligned currents flowing out from the northern ionosphere. Field-aligned currents for zero (nonzero) IMF $B_{x}$ and $B_{y}$ components are marked by the dashed (dotted) curves.

However, such effects exist (see, e.g. Iijima and Shibaji, 1987). The relationship of the NBZ-current distribution with IMF $B_{y}$ in the southern polar cap during northward IMF has been obtained using magnetic field data from the MAGSAT spacecraft firstly described by Iijima et al. (1984) and by the calculations using the spherical model (Alexeev and Belenkaya, 1985). The calculation results were in a good accordance with the observations. It was shown that the location of the NBZ-currents depends on $B_{y}$. This is why the Region 1 currents connected with NBZ-currents are also controlled by $B_{y}$ (the dependence of the field-aligned Region 1 currents on $B_{x}$ and $B_{y}$ was also studied by Belenkaya et al., 2004).

\section{Deformation of the Region 1 field-aligned current sheets in the transition current system due to the IMF}

Interplanetary magnetic field $(\boldsymbol{B})$ penetrating into the magnetosphere leads to the geomagnetic field line shifts (e.g. Belenkaya, 2002). The penetration of the interplanetary mag- netic and electric fields into the magnetosphere was studied by Alexeev (1986). A finite-conductivity solution for the magnetic field in the magnetosheath was constructed. Magnetic field diffusion caused by finite conductivity results in only a partial screening of the outer field in a dissipative layer near the magnetopause, with the residual magnetic field penetrating into the magnetosphere $(\boldsymbol{b})$. Dependence of the magnetic and electric fields at the magnetopause on the magnetic Reynolds number was determined.

The solution for $\boldsymbol{b}$ in the paraboloid magnetospheric model is a uniform field directed along the undisturbed IMF and equal to $\boldsymbol{b}=k \boldsymbol{B}$ (where $k$ is a coefficient of IMF penetration). Tsyganenko (2002) found the best correspondence between his model of the near-Earth magnetosphere and observational data for the coefficient of IMF penetration into the Earth's magnetosphere of between 0.15 and 0.8 . Here we investigate the dawn-dusk displacement of the transition current system magnetic field's extrema. Figure 2 shows that these extrema lie on the line perpendicular to the plane of the mostly strong field-aligned Region 1 currents. In Fig. 2 the current sheet with the field-aligned current flowing into (out of) the ionosphere is located at $6^{h}\left(18^{h}\right)$. The magnetic field of such a current system is southward at noon and northward at midnight. When these currents are deformed by the IMF, the line perpendicular to the surface containing them is shifted.

It was shown that the transition current system arises when IMF turns northward from the near horizontal direction at the front of the CME-encountered magnetosphere (Clauer et al., 2001; Belenkaya et al., 2004). The global magnetospheric configuration for northward IMF is mostly important for the forming of the transition current system. During northward IMF, two magnetic nulls, $O_{n}$ and $O_{s}$, arise inside the magnetosphere, near the cusp regions (see, e.g. Belenkaya, 1998a, b). The penetrated portion of IMF $b$ can provide the most influence on the geomagnetic field lines near the magnetic field neutral points $O_{n}$ and $O_{s}$ (see Fig. 1). As a result, it shifts magnetic nulls and field lines crossing them. The components of the vector of the neutral point displacement ( $d x_{0}$, $d y_{0}, d z_{0}$ ) are proportional to the corresponding components of the penetrated IMF $\boldsymbol{b}\left(b_{x}, b_{y}, b_{z}\right)$.

Figure 3 schematically presents the northern part of the transition current system, including the NBZ and Region 1 field-aligned currents closed by the ionospheric Pedersen currents. NBZ current flows into the ionosphere in the dusk, then flows on the ionosphere as the Pedersen current, which is closed later by the dusk field-aligned Region 1 current flowing out from the northern ionospheric open field line boundary (marked by a circle) to the southern neutral point. Then the Region 1 dawn current returns from the southern neutral point to the open field line boundary of the northern polar cap. After that it flows as the Pedersen ionospheric current, and is closed by the NBZ field-aligned current flowing out from the northern ionosphere.

Figure 3 represents the view from the Sun (dusk is on the right) for the case $b_{x}<0, b_{y}>0$, and $b_{z}>0$, when $O_{s}$ is shifted 
to the dawn-noon sector. Figure 4 illustrates this situation for the 31 March 2001 event. Calculations are performed in the paraboloid model of the Earth's magnetosphere described, for example, in Alexeev et al. (2003). When $b_{x}>0, b_{y}>0$, and $b_{z}>0, O_{s}$ is shifted to the dawn-night sector.

Figure 5 shows schematically the projection on the Earth's equatorial plane of the line $X^{\prime}$ perpendicular to the surface containing the deformed Region 1 currents $(\eta)$. As it was mentioned earlier, this line coincides with the direction to the transition current system magnetic field extrema. In Fig. 5, line $O O_{s}^{\prime \prime}$ marks a direction parallel to the projection on the equatorial plane of a line belonging to the surface $\eta$ and crossing the southern neutral point $O_{s}^{\prime}$ and the projection on this surface of the Earth's center $(O)$. $O O_{s}^{\prime \prime}$ is perpendicular to $X^{\prime}$. When $b_{x}<0, b_{y}>0$, and $b_{z}>0, O_{s}^{\prime}$ is shifted to the dawn-noon sector relative to its location for the zero IMF $\left(O_{s}\right)$. Figure $5 a$ represents the case when $b_{x}<0, b_{y}>0$, and $b_{z}>0$, and Fig. 5b corresponds to $b_{x}>0, b_{y}>0$, and $b_{z}>0$. For the last case, $O_{s}^{\prime}$ is shifted to the dawn-night sector relative to $O_{s}$, and $X^{\prime}$ points to the dawn-noon.

The angle $\gamma$ between $X^{\prime}$ and $X$ (new and old directions to the minimum magnetic field of the transition current system) is determined by the equation:

$\tan \gamma=\frac{y}{x}=-\frac{b_{y}}{b_{x}}$,

because displacements are caused by the corresponding IMF components, and the minus sign reflects the chosen sense of the angle $\gamma$ measurement: it is positive in a direction from noon to dusk. When the signs of $b_{x}$ and $b_{y}$ are different, the minus sign in the right side of Eq. (4) provides the positive value of $\gamma$, in accordance with Fig. 5a. When the signs of $b_{x}$ and $b_{y}$ are the same, the minus sign in Eq. (4) describes the position of $X^{\prime}$ corresponding to Fig. 5b. The displacements of the transition current system magnetic field extrema will be determined for each of the events studied by us previously (Clauer et al., 2001; Belenkaya et al., 2004).

In Fig. 3, location of the southern neutral point $O_{s}$ for IMF $B_{x}$ and $B_{y}$ components equal to zero (with $B_{z}>0$ ) is shown together with the corresponding Region 1 currents intersecting it (dashed curves). If for northward IMF, the $B_{x}$ and $B_{y}$ components are not equal to zero, their penetration into the magnetosphere causes a shift in the magnetic field lines. Field-aligned Region 1 currents which flow along these shifted field lines are marked by dotted curves. $O_{s}^{\prime}$ is a new (shifted) location of the southern neutral point for the nonzero IMF $B_{x}$ and $B_{y}$ components.

\section{$3.1 \quad 24-27$ September 1998 storm}

The geomagnetic storm on 24-27 September 1998, was initiated in response to the sudden solar wind dynamic pressure pulse (at 23:45 UT on 24 September). Simultaneous with the pressure increase, the IMF became northward. Figures 6
31.03.2001

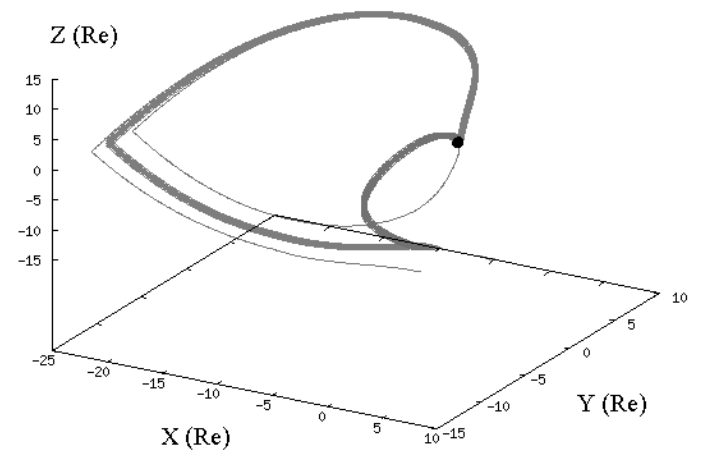

Fig. 4. Magnetic field lines (bold curves) going from the northern polar cap boundary to the southern magnetic field neutral point calculated in the paraboloid model of the terrestrial magnetosphere. The closest to the bold line going through the tail closed and open field lines are marked by thin curves. $X, Y$, and $Z$ are the axes in the solar-magnetospheric coordinate system; distances along them are measured in the Earth's radii $\left(R_{E}\right)$. Calculations are performed for the 31 March 2001 event.

a)

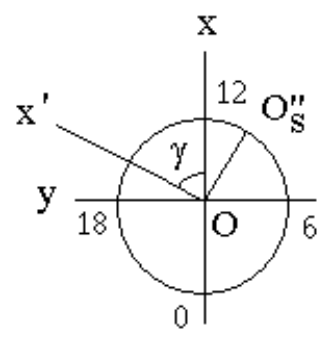

b)

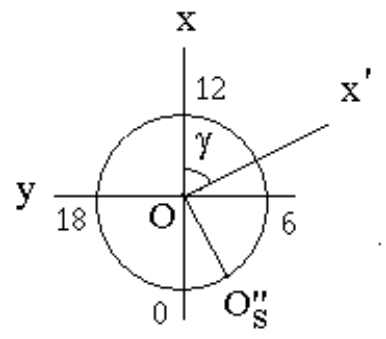

Fig. 5. Projection on the Earth's equatorial plane of the $X^{\prime}$ line perpendicular to the surface $(\eta)$ containing the Region 1 currents deformed by the IMF. The transition current system is magnetic field extrema are located along this line. $O O_{S}^{\prime \prime}$ marks a direction parallel to the projection on the equatorial plane of a line belonging to the surface $\eta$ and crossing the southern neutral point $O_{S}^{\prime}$ and the projection on this surface of the Earth's center $(O)$. Components of the magnetic field penetrated into the magnetosphere from the solar wind: (a) $b_{x}<0 ; b_{y}>0 ; b_{z}>0$; (b) $b_{x}>0 ; b_{y}>0 ; b_{z}>0$. 


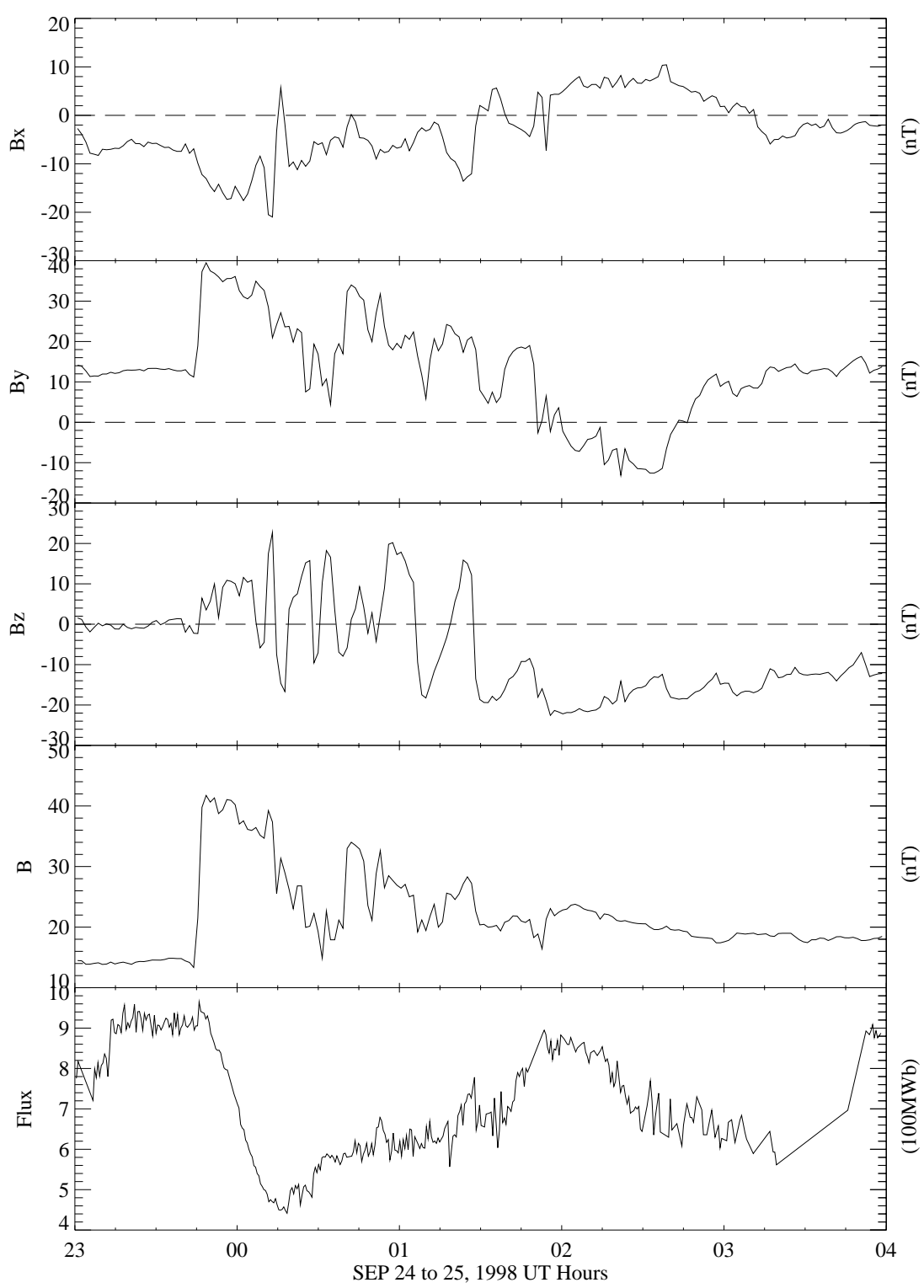

Fig. 6. Solar wind parameters offset by $24 \mathrm{~min}$ on $24-25$ September 1998 . Observations by Wind/MFI of the solar wind magnetic field in the GSM coordinates. From the top to bottom: $B_{x}, B_{y}, B_{z}, B$, and polar cap magnetic flux, which decreased due to northward IMF rotation.

and 7, taken from Clauer et al. (2001), show the solar wind parameters for this case. From Fig. 6 it is seen that just after the interplanetary shock front passage, the IMF components became: $B_{x}=-18 \mathrm{nT}, B_{y}=40 \mathrm{nT}, B_{z}=10 \mathrm{nT}$ (before the front the IMF components were $B_{x}=-10 \mathrm{nT}, B_{y}=10 \mathrm{nT}$, $B_{z}=0 \mathrm{nT}$ ). For calculation of the transition current system's magnetic field, only the IMF components after the interplanetary shock front passage are significant, as before the front $B_{z}$ was near zero, and no transition current system existed. It arose only after the IMF rotated northward at the CME front.

As $b_{x}<0$ and $b_{y}>0$, the southern neutral point $O_{s}$ is shifted to the dawn-noon sector. Thus, the current circuit of the northern part of the transition current system located in the dawn-dusk plane for $b_{x}=0, b_{y}=0$, and $b_{z}>0$, becomes deformed under the action of the radial and azimuthal components of the northward IMF (see Fig. 3). This deformation creates a displacement of its magnetic field extrema. For example, the maximum southward magnetic field of the transition current system is shifted from the noon to dusk on the angle $\gamma$. The maximum northward magnetic field is shifted from the midnight to dawn on the same angle. For this case, $\tan \gamma=-b_{y} / b_{x}=-B_{y} / B_{x}=40 \mathrm{nT} / 18 \mathrm{nT}=2.2$, and $\gamma=66^{\circ}$. Thus, $\gamma$ describing the azimuthal shift of the magnetic field northward and southward disturbances will be of the order of $66^{\circ}=4.4 \mathrm{~h}$. 


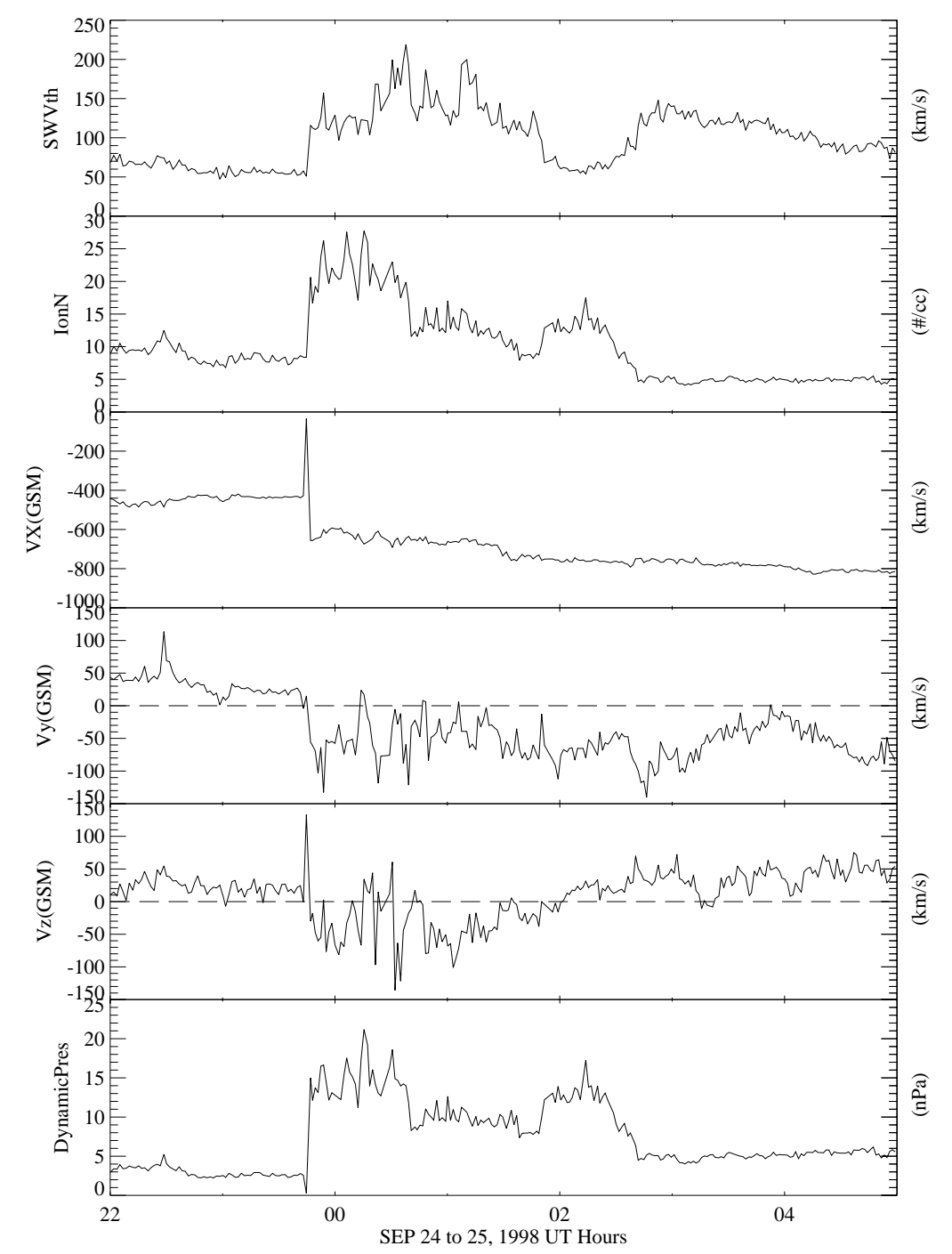

Fig. 7. Solar wind parameters offset by $24 \mathrm{~min}$ on 24-25 September 1998. Observations by Wind/SWE of the solar wind (top to bottom) of the thermal velocity, $V_{t h}$; solar wind ion density, $N$; solar wind bulk velocity, $V$, in the GSM coordinates; and solar wind dynamic pressure, $p=n V_{x}^{2}$.

Figure 8 presents a Magnetic Local Time (MLT) versus Universal Time (UT) map showing the $H$ component magnetic field disturbance obtained from middle- and lowlatitude ground-based magnetometers on 24-25 September 1998 (Clauer et al., 2001). The contours show a magnetic disturbance at 25-nT intervals from the quiet-day variation, with positive perturbations shaded in blue and negative ones shaded in red. We see that at 23:45 UT on 24 September the nightside enhancement of the northward magnetic field $(>50 \mathrm{nT})$ is observed to be located roughly from $23: 50 \mathrm{~h}$ MLT to 04:50 $\mathrm{h}$ MLT (at 02:50 $\mathrm{h} \mathrm{MLT,} \mathrm{on} \mathrm{average).} \mathrm{The}$ dayside enhancement of the southward magnetic field dis- turbance $(<50 \mathrm{nT})$ is observed between $\sim 14.2 \mathrm{~h}$ MLT and $20.2 \mathrm{~h}$ MLT (at 17:00 h MLT, on average). Thus, for very crude estimations, we can take the nightside shift of the northward magnetic perturbations to be $\sim 2.5 \mathrm{~h}$ from midnight to dawn, and the dayside shift of the southward magnetic disturbances to be $\sim 5 \mathrm{~h}$ from noon to dusk, with the average value of the dawn-dusk asymmetry of the order of $(2.5+5) / 2 \sim 3.8 \mathrm{~h}$. Comparison with our rough calculations $(\gamma=4.4 \mathrm{~h})$ gives a crude accordance of the value and the shift direction of the observed dawn-dusk asymmetry of the lowlatitude magnetic field disturbances interpreted by us as a transition current system magnetic field. 


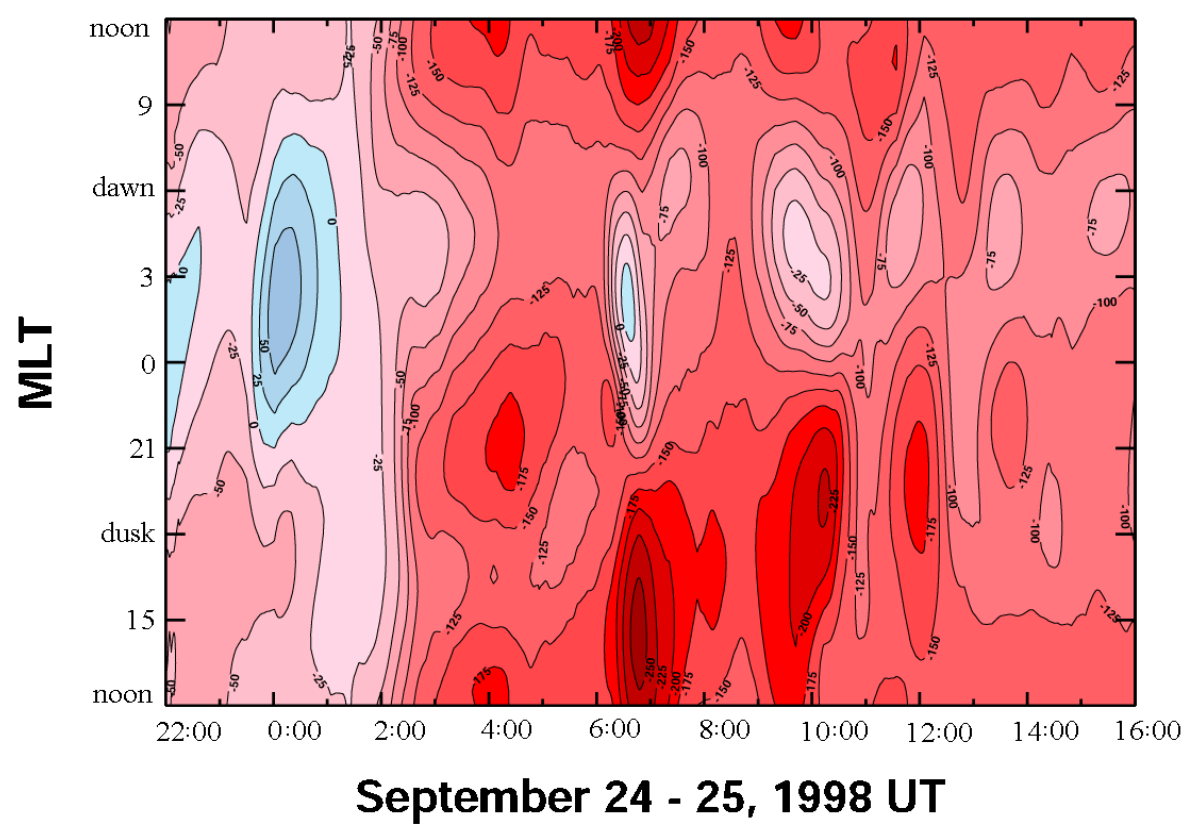

Fig. 8. Magnetic Local Time (MLT) versus Universal Time (UT) map showing the $H$ component of the magnetic disturbance field obtained from middle- and low-latitude ground-based magnetometers on 24-25 September 1998. The effect from a quiet day (17 August) variation is removed. The contours show a magnetic disturbance at 25-nT intervals from the quiet-day variation, with positive perturbations shaded in blue and negative ones shaded in red.

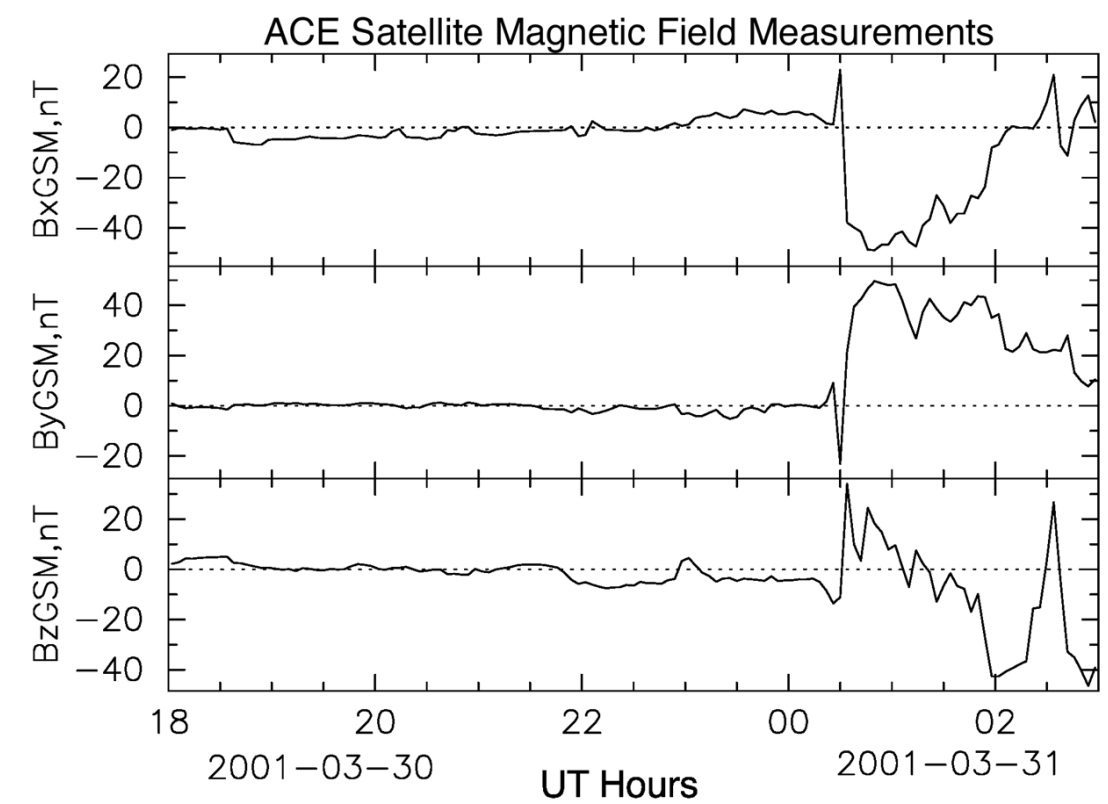

Fig. 9. Solar wind magnetic field components $B_{x}, B_{y}$, and $B_{z}$ in the GSM coordinates measured at the ACE spacecraft. Horizontal axis shows UT hours from 18:00 UT on 30 March to 03:00 UT on 31 March. 


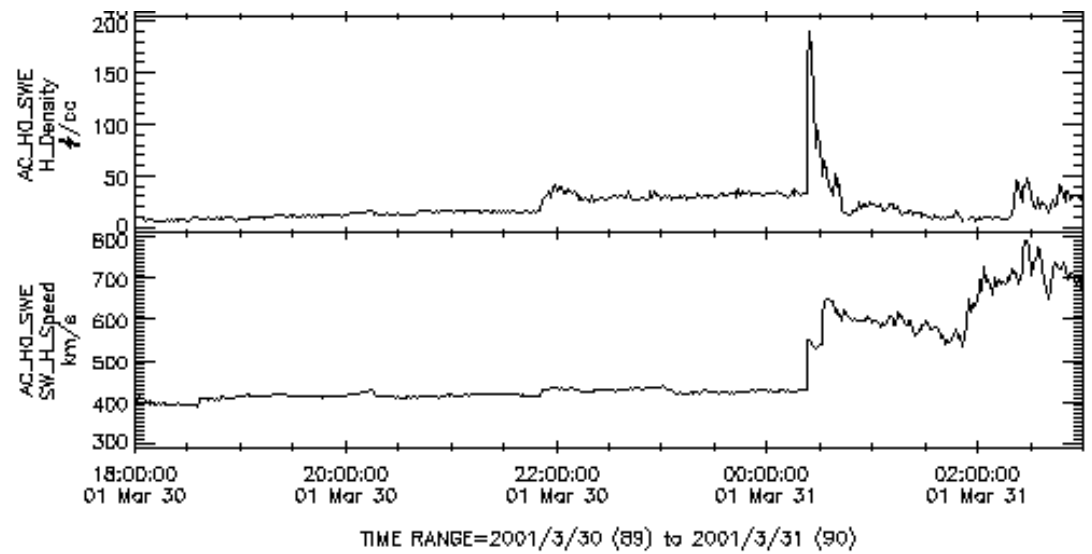

acknowledge data provider(s), N. Ness at Bartol Research Institute and D. J. WoComos at SWRI and CCAWeb when using thes Generated by CLAiWeb on Thu Apr 12 07:27:35 2007

Fig. 10. Solar wind density and velocity values in the GSM coordinates measured at the ACE spacecraft. Horizontal axis shows UT hours from 18:00 UT on 30 March to 03:00 UT on 31 March.

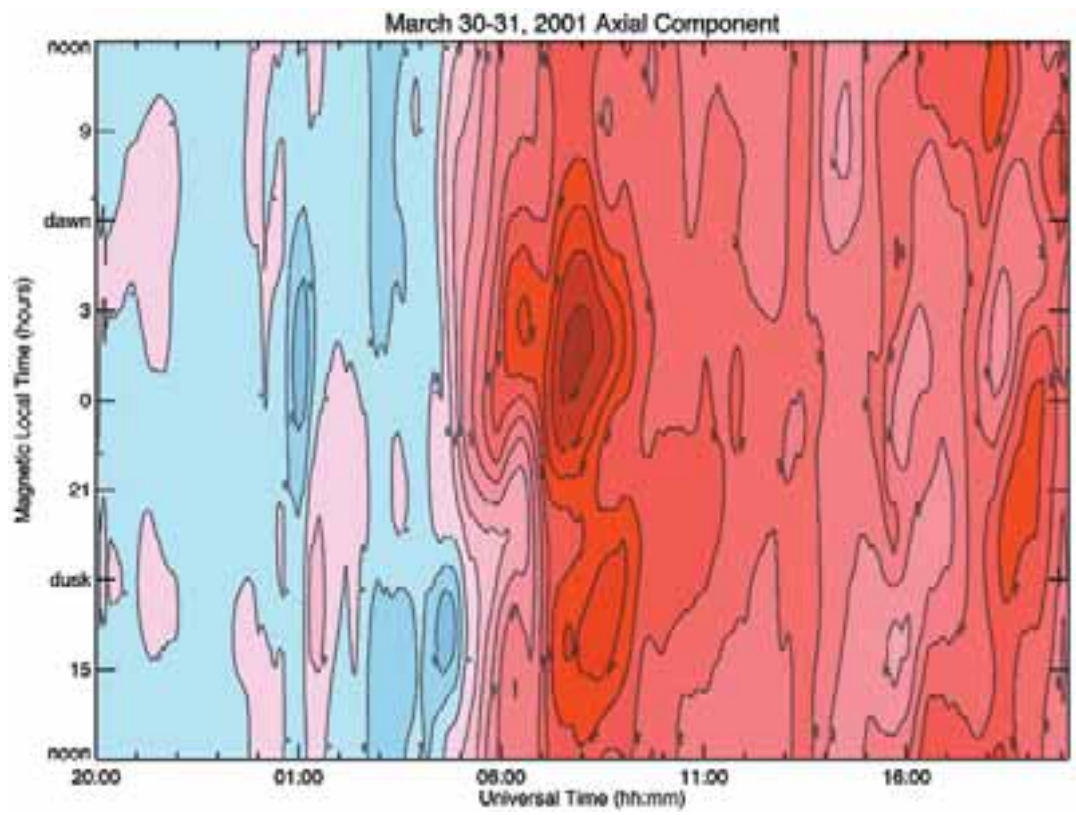

Fig. 11. A MLT-UT map of the $H$ component of the world-wide magnetic disturbance field measured by 19 low-latitude ground magnetic observatories from 20:00 UT on 30 March 2001 to 20:00 UT on 31 March 2001. The quiet-time field has been removed from the data using observations from the quiet day 15 March 2001. The contour step is $50 \mathrm{nT}$, with blue indicating positive disturbances and red negative disturbances (Skoug et al., 2003).

\subsection{March 2001 event}

A large coronal mass ejection encountered the Earth's magnetosphere at 00:51 UT on 31 March 2001 (see Figs. 9 and 10 representing the ACE satellite measurements). Just behind the front of the CME the averaged values of the IMF components were: $B_{x}=-40 \mathrm{nT}, B_{y}=40 \mathrm{nT}$, and $B_{z} \approx 30 \mathrm{nT}$. As again $b_{x}<0$ and $b_{y}>0$ for $b_{z}>0$, the southern neutral point $O_{s}$ is shifted to the dawn-noon sector, and the maximum northward (southward) magnetic field of the transition current system should be shifted from midnight (noon) to dawn (dusk) on the angle $\gamma$. The value of $\tan \gamma=-B_{y} / B_{x}=40 \mathrm{nT} / 40 \mathrm{nT}=1$, and $\gamma=45^{\circ}$, which corresponds to $3 \mathrm{~h}$.

Figure 11 (Skoug et al., 2003) shows the MLT-UT map for this case, which demonstrates the northward disturbance ( $>100 \mathrm{nT}$ ) located from 23:00 h to 04:00 $\mathrm{h}$ MLT (on average, 


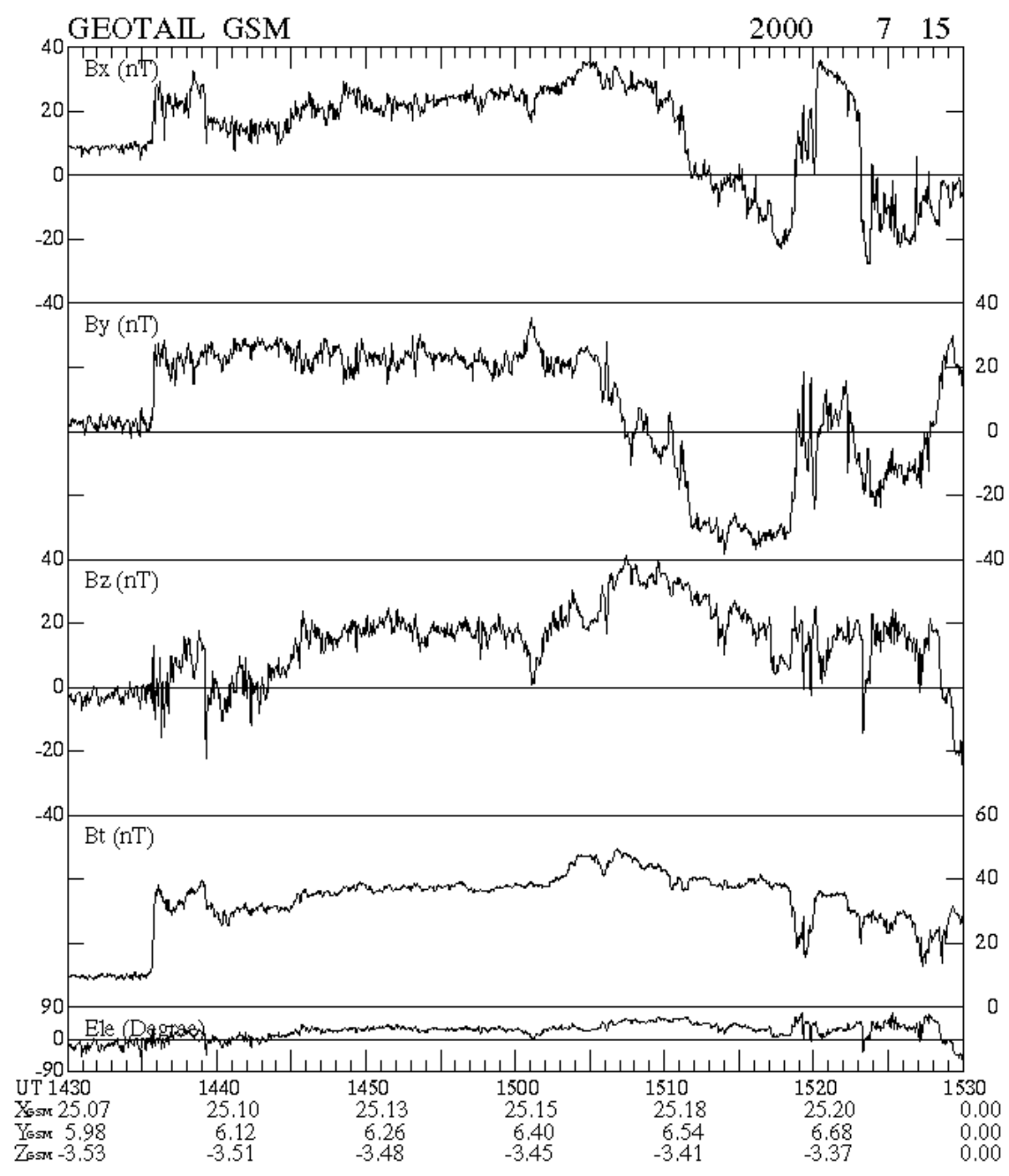

Fig. 12. The IMF components $\left(B_{x}, B_{y}\right.$, and $\left.B_{z}\right)$ and value of the total magnetic field $(\boldsymbol{B})$ as measured by the Geotail satellite on 15 July 2000.

at 02:50 h MLT), while the southward disturbance was absent (near zero) at this time. Thus, for a crude estimation, we take the nightside shift of maximum northward magnetic field perturbations to be $\sim 2.5 \mathrm{~h}$ from midnight to dawn, which can be considered as the average value of the dawn-dusk magnetic disturbance asymmetry. Comparison with our rough calculations $(\gamma=3 \mathrm{~h})$ gives a good agreement.

\subsection{The Bastille day storm of 15-16 July 2000}

Figures 12 and 13 demonstrate the above described conditions in the solar wind measured by the Geotail satellite: almost a simultaneous increase in the dynamic pressure and northward IMF turning occurred at $\sim 14: 35-14: 36$ UT on 15 July 2000. For this event $B_{x}=30 \mathrm{nT}, B_{y}=30 \mathrm{nT}$, and $B_{z}=15 \mathrm{nT}$ (see Fig. 12). Thus, $\tan \gamma$ is equal to
$-30 \mathrm{nT} / 30 \mathrm{nT}=-1$, and $\gamma$ is equal to $-45^{\circ}$, or $-3 \mathrm{~h}$. In this case $b_{x}>0$, and $b_{y}>0$, correspondingly, and $O_{s}$ is displaced to the night-dawn sector. According to Fig. 5b, the line of the maximum southward magnetic field of the transition current system $\left(X^{\prime}\right)$ is rotated from the noon direction on the angle $\gamma$, which in this case is negative. Thus, the southward disturbance should be shifted from noon to dawn. The direction of the shift for this case is opposite relative to those in the previous two events characterized by negative $b_{x}$. The maximum northward magnetic disturbance should be shifted by angle $\gamma$ from midnight to dusk.

Figure 14 shows the low-latitude ground disturbances of the $H$ component magnetic field (the MLT-UT map) for 15 July 2000 (the 14:30 UT profile is subtracted). We see that the most intense northward perturbations are located between 17:00 $\mathrm{h}$ and 01:00 $\mathrm{h}$ MLT, with the average position at 


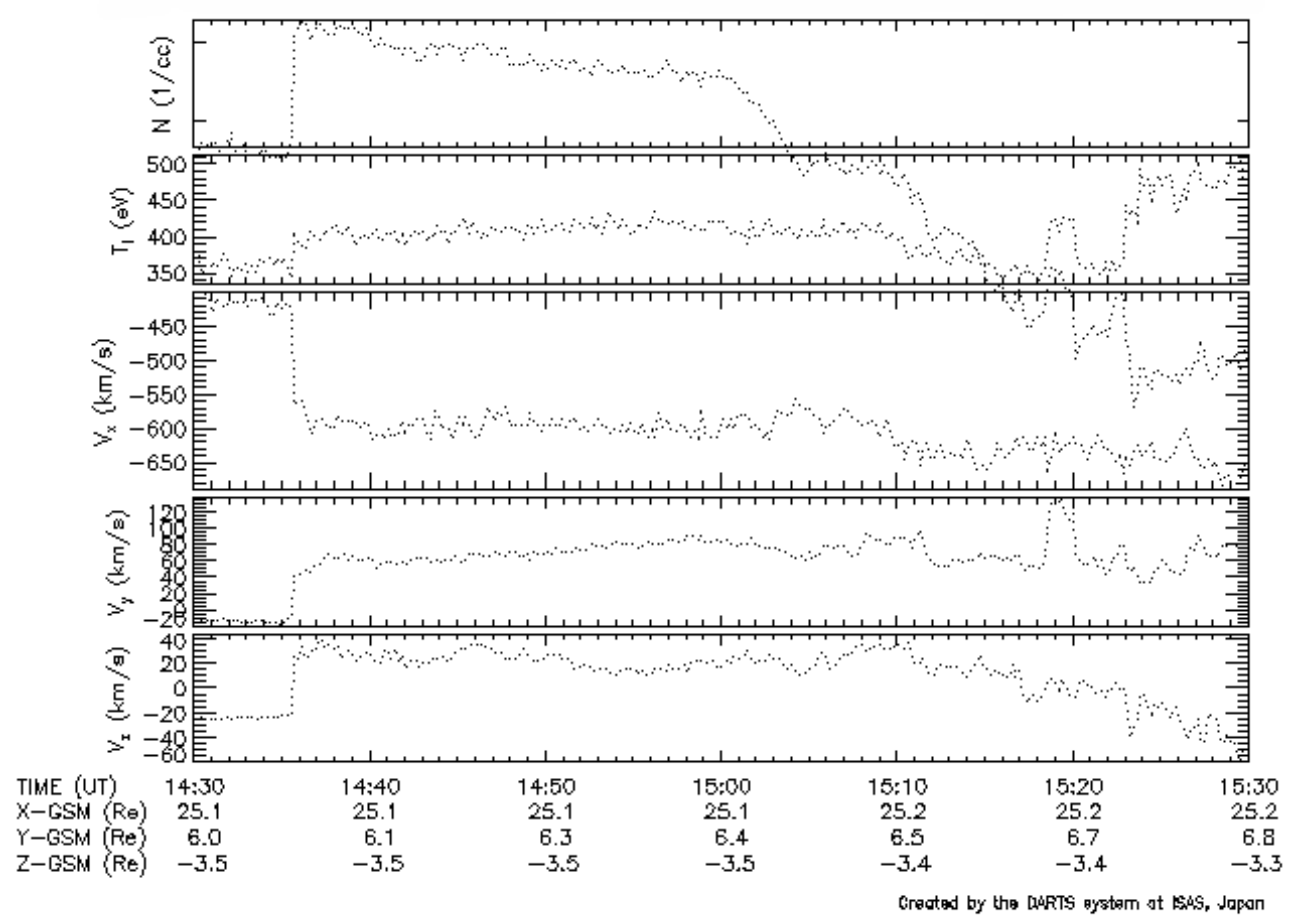

Fig. 13. Solar wind parameters measured by the Geotail satellite on 15 July 2000. From the top to bottom; density; temperature; $x, y$, and $z$-components of velocity.

21:00 $\mathrm{h}$ MLT. The corresponding shift in the "nightside magnetic field effect" of the transition current system from midnight to dusk is equal to $-3 \mathrm{~h}$. The southward perturbations are very weak in comparison with the nightside data. They are located between 07:00 $\mathrm{h}$ and 09:00 $\mathrm{h}$ MLT, with the average position at 08:00 $\mathrm{h}$ MLT. In spite that their value is near zero, we take them into account, because the location of such type of disturbance is identified in this case. The corresponding shift in the "dayside magnetic field effect" of the transition current system from noon to dawn is equal to $-4 \mathrm{~h}$. The averaged observed dawn-dusk asymmetry can be described by $-(3+4) / 2=-3.5 \mathrm{~h}$, which is in good accordance with our crude calculations $(-3 \mathrm{~h})$.

Thus, using a very simplified model for the magnetic field of the transition current system, we explained the observed dawn-dusk asymmetry of the low-latitude magnetic disturbances. We showed that $B_{x}$ and $B_{y}$ IMF components could be responsible for this asymmetry. We received good accordance of our estimations with observations of the directions and values of the transition current system magnetic field extrema displacements due to the IMF. The difference between the calculated and observed values of these displacements is $\leq 0.6 \mathrm{~h}$ for the studied events.

\section{Discussion}

It is necessary to note that processes described here should be considered during the characteristic time, which has a lower limit of the order of $10 \mathrm{~min}$ and the upper limit $\sim 30-40 \mathrm{~min}$. The upper limit $\left(t_{r}\right)$ is explained in the paper by Clauer et al. (2001), and this explanation is repeated for self-consistency in the present paper. The lower limit $\left(t_{l}\right)$ is determined by the exclusion from our consideration of the wave processes arising just after the interplanetary shock arrival. For example, Fujita et al. (2003) analyzed, in detail, the main impulse phase of the sudden commencement in the first 9 min after the interplanetary shock encounter with the magnetopause, and described two successive current systems generated by the pressure enhancement. During the time period $t_{l}$, a lot of processes connected with the magnetospheric compression disturbances occur. After the time period comparable with $t_{l}$, the magnetosphere reaches a new equilibrium state, corresponding to an increased solar wind pressure value. Our consideration works following this moment in time. Thus, we considered neither any wave processes nor the processes connected with the compression propagation. The other specific distinction of our approach is that we describe a peculiar situation when the interplanetary shock arrival is accompanied by the northward IMF rotation from the near horizontal direction. In many other studies, where 


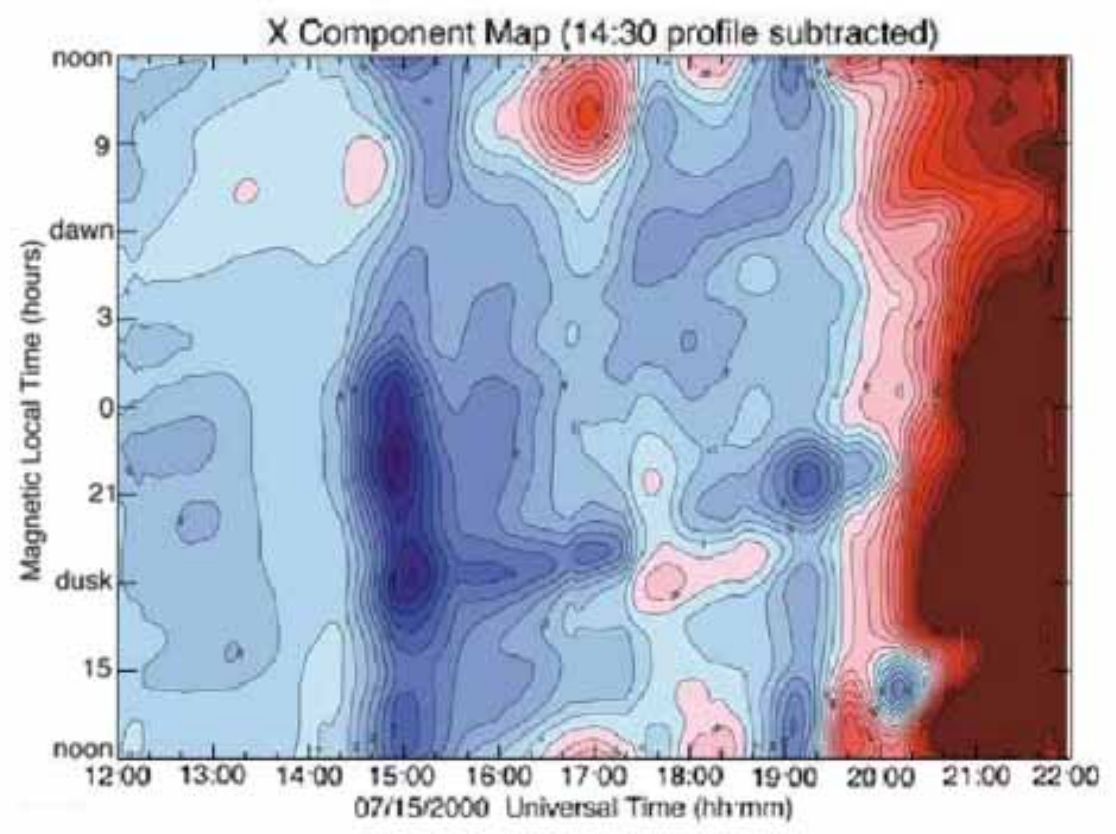

Fig. 14. Magnetic Local Time (MLT) versus Universal Time (UT) map showing the $H$-component magnetic disturbance field obtained from middle- and low-latitude ground-based magnetometers on 15 July 2000. The contours show a magnetic disturbance at 19-nT intervals with the subtracted quiet time variations (at 14:30 UT), with positive perturbations shaded in blue and negative ones shaded in red.

the magnetospheric response to the solar wind pressure pulse for northward IMF is investigated (e.g. Russell et al., 1994; Fujita et al., 2003), the interplanetary magnetic field does not change its orientation at the shock front. Under such circumstances, other kinds of transient current systems arise, different from the transition current system considered here.

With the help of a magnetohydrodynamic (MHD) model Kullen and Janhunen (2004) investigated how the large-scale topology of the magnetosphere develops for a constant IMF with different IMF clock angles. The main attention was given to the topological changes in the tail and the ionosphere. The authors noted that the field-aligned current pattern was for all MHD runs nearly unchanged, which does not allow us to compare our results with these MHD simulations. Tanaka et al. (2004), using a MHD simulation, investigated the formation of theta aurora under conditions of northward IMF with a great magnitude and the sign change of IMF $B_{y}$. The reconstruction of the magnetosphere/ionosphere system under such conditions differs significantly from the situation studied here, which also does not allow for any comparison with our results.

\section{Conclusions}

Here we consider a possible reason of the dawn-dusk asymmetry of the on-ground magnetic field created by the transition current system at low latitudes. The main contribution to this magnetic field is the formation of the Region 1 currents into a the three-dimensional high-latitude temporary existing transition current system. We showed previously that these currents provide the day-night asymmetry of the transition current system's magnetic field observed by the lowlatitude on-ground magnetometers under corresponding conditions in the solar wind: the almost simultaneous increase in the dynamic pressure and the northward rotation of the IMF from the near horizontal orientation (Clauer et al., 2001; Belenkaya et al., 2004). In the present paper we pay attention to the shift in the magnetospheric field lines (mostly significant in the vicinity of the magnetic field neutral points), which causes the displacement of the Region 1 current circuit.

We showed that due to $B_{x}<0$, and $B_{y}>0$, the northern Region 1 current circuit is shifted to the dawn-noon sector, and respectively, the northward (southward) low-latitude magnetic field perturbation should be shifted from midnight (noon) to dawn (dusk). Oppositely, for $B_{x}>0$, and $B_{y}>0$, the Region 1 current circuit is shifted to the dawn-nightside sector. As a result, the northward (southward) low-latitude magnetic field perturbation should be shifted from midnight (noon) to dusk (dawn).

The obtained results were compared with the available observations. The direction of the dawn-dusk asymmetry of the low-latitude on-ground magnetic field disturbances received from the applied simple model, coincides with the magnetometers data. The value of an angle, which measures this asymmetry, coincides with the observed one, with an accuracy of $\pm 0.6 \mathrm{~h}$, which gives good accordance for such rough estimations. 
Acknowledgements. We thank the ACE and Geotail mission teams, J. H. King at NSSDC and NASA's GSFC and CDAWeb for providing the solar wind and IMF parameters. We thank the ACE data providers N. Ness at Bartol Research Institute, and D. J. McComas at SWRI. We also thank S. Kokubun at STELAB Nagoya University and T. Nagai at Tokyo Institute of Technology, and the DARTS system in ISAS, Japan for providing the Geotail GSM IMF data. A portion of this research has been supported by the National Science Foundation Grant OPP-0220735 and NASA grant NAG5-12176. This work was partially supported by the INTAS Grant No 03-513922 and by the RFBR Grants 05-05-64435 and 07-05-00529.

Topical Editor I. A. Daglis thanks S. Eriksson and S. Fujita for their help in evaluating this paper.

\section{References}

Alexeev, I. I.: The penetration of interplanetary magnetic and electric fields onto the magnetosphere, J. Geomag. Geoelectr., 38, 1199-1221, 1986.

Alexeev, I. I. and Belenkaya, E. S.: Convection of the magnetospheric plasma on the open field line region, Geomagn. Aeron., 25, N 3, 450-457, 1985 (in Russian).

Alexeev, I. I., Belenkaya, E. S., Bobrovnikov, S. Y., and Kalegaev, V. V.: Modelling of the electromagnetic field in the interplanetary space and in the Earth's magnetosphere, Space Science Reviews, 107(1-2), 7-26, 2003.

Belenkaya, E. S.: Reconnection modes for near-radial IMF, J. Geophysical Res., 103, No A11, 26 487-26494, 1998a.

Belenkaya, E. S.: High-latitude ionospheric convection patterns dependent on the variable IMF orientation, J. Atmos. SolarTerrestrial Phys., 60/13, 1343-1354, 1998b.

Belenkaya, E. S.: Influence of the interplanetary magnetic field on the magnetosphere forming, Itogy Nauki i Techniki. Issledovanie Kosmicheskogo prostranstva, 33a, 233 p., VINITI, Moscow, 2002 (in Russian).

Belenkaya, E. S., Alexeev, I. I., and Clauer, C. R.: Field-aligned current distribution in the transition current system, J. Geophys. Res., 109, A11207, doi:10.1029/2004JA010484, 2004.

Blomberg, L. G., Cumnock, J. A., Alexeev, I. I., Belenkaya, E. S., Bobrovnikov, S. Yu., and Kalegaev, V. V.: Transpolar aurora: Time evolution, associated convection patterns, and a possible cause, Ann. Geophys., 23, 1917-1930, 2005.
Clauer Jr., C. R., Alexeev, I. I., Belenkaya, E. S., and Baker, J. B.: Special features of the September 24-27, 1998 storm during high solar wind dynamic pressure and northward interplanetary magnetic field, J. Geophys. Res., 106(A11), 25 695-25 712, 2001.

Fujita, S., Tanaka, T., Kikuchi, T., Fujimoto, K., and Itonaga, M.: A numerical simulation of the geomagnetic sudden commencement: 2. Plasma processes in the main impulse, J. Geophys. Res., 108(A12), 1417, doi:10.1029/2002JA009763, 2003.

Fukushima, N.: Equivalence in ground geomagnetic effect of Chapman-Vestine's and Birkeland-Alfven's electric currentsystems for polar magnetic storms, Rep. Ionos. Space Res. Jpn., 23, 219, 1969.

Iijima, T., Potemra, T. A., and Zanetti, L. J.: Large-scale Birkeland currents in the dayside polar region during strongly northward IMF; A new Birkeland current system, J. Geophys. Res., 89(9), 7441-7452, 1984.

Iijima, T. and Shibaji, T.: Global characterisics of northward IMF - associated (NBZ) field-aligned currents, J. Geophys. Res., 92, 2408-2424, 1987.

Kullen, A. and Janhunen, P.: Relation of polar auroral arcs to magnetotail twisting and IMF rotation: a Systematic MHD simulation study, Ann. Geophys., 22, 951-970, 2004.

Russell, C. T., Ginskey, M., and Petrinec, S. M.: Sudden impulses at low-latitude stations: Steady state response for northward interplanetary magnetic field, J. Geophys. Res., 99, 253-261, 1994.

Siscoe, G. L., Erickson, G. M., Sonnerup, B. U. O., Maynard, N. C., Siebert, K. D., Weimer, D. R., and White, W. W.: Global role of $E_{\|}$in magnetopause reconnection: An explicit demonstration, J. Geophys. Res., 106, A7, 13 015-13 022, 2001.

Skoug, R. M., Thomsen, M. F., Henderson, M. G., Funsten, H. O., Reeves, G. D., Pollock, C. J., Jahn, J.-M., McComas, D. J., Mitchell, D. G., Brandt, P. C., Sandel, B. R., Clauer, C. R., and Singer, H. J.: Tail-dominant storm main phase: 31 March 2001, J. Geophys. Res., 108(A6), 1259, doi:10.1029/2002JA009705, 2003.

Tanaka, T., Obara, T., Kunitake, M., Formation of the theta aurora by a transient convection during northward interplanetary magnetic field, J. Geophys. Res., 109, A09201, doi:10.1029/2003JA010271, 2004.

Tsyganenko, N. A.: A model of the near magnetosphere with a dawn-dusk asymmetry. Mathematical structure, J. Geophys. Res., 107(A8), 10.1029/2001JA000219, 2002. 